\title{
Interaction between solitons in gauge theories
}

\author{
M. Baker \\ University of Washington, Seattle, Washington 98195 \\ J. S. Ball \\ University of Utah, Salt Lake City, Utah 84112 \\ F. Zachariasen \\ California Institute of Technology, Pasadena, California 91125
}

(Received 17 March 1986)

\begin{abstract}
A systematic method for obtaining asymptotic multisoliton solutions in gauge theories is given. These solutions are used to investigate the interaction between vortex lines in type-I and type-II superconductors, reproducing the known behavior. The application to QCD flux tubes and glueballs obtained from the long-range effective Lagrangian yield the following results: (1) No long-range Van der Waals-type forces exist between these solitons in spite of the fact that the Abelian force law obtained from this model is a linear potential; (2) the interactions between flux tubes and between flux tubes and anti-flux-tubes are identical, being repulsive at long range and strongly attractive at short range. This behavior differs sharply from the superconductor case, and results from the differences between the gauge groups $S U(2)$ and $U(1)$.
\end{abstract}

\section{INTRODUCTION}

Strong interactions are short range, yet perturbative QCD predicts long-range forces between color-neutral states-hadrons-just as electrodynamics does. Evidently nonperturbative effects in QCD at long range mask these forces; the long-range confining force between colored objects (which is also nonperturbative) has no Van der Waals-type force between colorless objects associated with it.

In phenomenological models of confinement, such as bag models, ${ }^{1}$ long-range forces between hadrons are simply decreed not to exist. According to these models, no fields extend beyond the bag boundary, and therefore no interaction is possible there. But bag models have not yet been convincingly derived from $\mathrm{QCD}$, so the mechanism by which fields are confined to the bag is not fully understood. Ferroelectric ${ }^{2}$ models of confinement come closest to providing this understanding. These are models which postulate a $Q C D$ vacuum with a field-dependent dielectric constant which vanishes at some nonzero color-electric field, just as in a classical ferroelectric. The bag boundary is supposed to be defined by this value of the electric field. Outside, the dielectric constant, and consequently the electric displacement, vanishes; also, no color-magnetic fields are present. The one-loop QCD dielectric constant at short range has this property; ${ }^{2}$ the assumption in this picture is either that the short-range value can also be used at long range, or that the long-range dielectric constant may differ, but still acts like a ferroelectric.

An alternative picture of confinement is that of a dual superconductor. ${ }^{3}$ Here the bag model is simply an approximation to the exact field equations, and it is not true that fields are entirely confined within the bag. The bag is not a region outside of which the dielectric constant vanishes; it is rather a region inside of which a Higgs potential becomes small. The primordial version of this picture is the relativistic superconductor, or Abelian Higgs model $^{4}$ (in the QCD version the electric and magnetic fields are interchanged). The bag is a region of normal vacuum within the superconducting vacuum produced by the introduction of sources (monopoles in the relativistic superconductor; quarks in QCD) via the Meissner effect. The bag boundary is not sharp; there is a smooth transition from the perturbative vacuum to the superconducting vacuum. All fields are continuous, and as they penetrate the superconducting vacuum they approach their asymptotic values exponentially.

In this picture the absence of long-range forces between colorless excitations is not obvious. The field equations are nonlinear, and the asymptotic values of the fields are not, in general, zero. To understand what long-range forces are in fact present requires a careful analysis of the field equations. It is this which we wish to report here.

We will study both the relativistic superconductor, and the effective action we have recently suggested as a description of long-range Yang-Mills theory. 5 In both of these cases the excitations in question are soliton solutions of classical nonlinear field equations. In each case the solitons contain long-range fields, although quantities of physical interest, such as the energy density, fall off exponentially with distance. Our conclusions are, first, that multisoliton solutions to the nonlinear equations can be constructed and written down analytically in the limit of large soliton separation, and, second, that the interaction energy between solitons falls exponentially with separation. Thus, no long-range forces exist.

For the relativistic superconductor, our approach reproduces the well-known results ${ }^{6}$ that in a type-I superconductor parallel flux tubes attract, while in type II they re- 
pel. Asymptotically, the interaction energy is, in both cases, just the sum of the energies of the individual flux tubes. At zero separation, in type I the energy of a twounit vortex is less than that of two one-unit vortices; in type II it is greater.

In the QCD dual superconductor, the situation is very different. Here, as the separation between flux tubes decreases, the interaction energy rises to perhaps 5-10\% above its asymptotic value, then falls to zero at zero separation. The basic reason for this fall is that in the non-Abelian SU(2) case which we discuss, a flux tube with two units of flux is topologically equivalent to zero units of flux-that is to the vacuum-in complete contrast to the ordinary superconductor. Therefore in QCD there is a potential barrier, outside of an attractive well, in the potential between two flux tubes. There are evidently a number of interesting phenomenological implications of this; some of these are discussed in Sec. V.

The organization of this paper will be as follows. In Sec. II a general superposition method for constructing multisoliton solutions is illustrated by its application to the familiar Landau-Ginsberg vortices of superconductivity. Section III contains the application of this general method to Yang-Mills flux tubes, while in Sec. IV we treat the glueball case. ${ }^{7}$

\section{SUPERPOSITION OF GAUGE SOLITONS}

The solitons which we will consider have the following properties: (1) For large distances from the origin (the center of the soliton), the vector potential is proportional to $1 / r$ and (2) some other field or fields are constant. The required property is that the asymptotic fields are gauge equivalent to the vacuum.

The superposition of such solitons can be accomplished as follows. Transform each soliton solution to the gauge in which the vector potential vanishes exponentially at large distance (this gauge transformation is in general singular). In this gauge, the fields that go to a constant asymptotically will approach their constant value exponentially. One can now simply superimpose the exponential parts of the fields for each soliton. This clearly satisfies the asymptotic field equations, as they are linear. Further, as one approaches the center of a particular soliton, the local fields differ from the single soliton solution only by the exponentially small fields due to the other solitons. Thus, when the solitons are widely separated we have an asymptotic multisoliton solution. Finally, after the superposition, an overall gauge transformation is made which removes the singularities at the origin of each soliton introduced by the singular gauge transformations performed at the beginning on the individual solitons.

As an illustration of this procedure we will construct an asymptotic multivortex-line solution to the LandauGinsberg equations of superconductivity. The vortex line is similar to the solitons which are of direct interest in the Yang-Mills case, but is much simpler, in that the theory is Abelian and only two fields are involved. We begin by briefly reviewing the superconducting vortex-line solution. The relevant fields are $A$, the vector potential, and $\Psi$, the order parameter (or scalar Higgs field in the Abelian
Higgs model version of the same theory ${ }^{4}$ ). The equations for a vortex line with one unit of flux are obtained by making the ansatz that in cylindrical coordinates $(r, \theta)$

$$
\Psi=\phi(r) e^{-i \theta},
$$

where $\phi$ is real. The resulting equations are

$$
\boldsymbol{\nabla} \times(\boldsymbol{\nabla} \times \mathbf{A})+\left(\mathbf{A}-\frac{\hat{\boldsymbol{\theta}}}{r}\right) \phi^{2}=0
$$

and

$$
-\nabla^{2} \phi-\phi+k^{2} \phi^{3}+\left|\mathbf{A}-\frac{\hat{\theta}}{r}\right|^{2} \phi=0 .
$$

Here, all constants have been scaled out and $k^{2}$ is the Landau-Ginsberg parameter. In the solution, the vector $\mathbf{A}$ is in the $\hat{\boldsymbol{\theta}}$ direction and goes to $\hat{\boldsymbol{\theta}} / r$ asymptotically, while $\phi$ goes to $\phi_{0}=1 / k$. Both $\mathbf{A}$ and $\phi$ vanish like $r$ as $r \rightarrow 0$. The complete asymptotic solution is

$$
\mathbf{A}=\left[\frac{1}{r}+a K_{1}\left(\frac{r}{k}\right)\right] \widehat{\boldsymbol{\theta}}
$$

and

$$
\phi=\frac{1}{k}+b K_{0}(\sqrt{2} r),
$$

where $K_{0}$ and $K_{1}$ are modified Bessel functions and $a$ and $b$ are constants that are determined by the solution.

To produce a multivortex solution we must first write $\mathbf{A}$ and $\Psi$ in a gauge in which $\mathbf{A}$ vanishes exponentially at large $r$. This is accomplished by the following gauge transformation:

$$
\Psi^{\prime}=e^{i \theta} \Psi \equiv \phi
$$

and

$$
\mathbf{A}^{\prime}=\mathbf{A}-\frac{\hat{\boldsymbol{\theta}}}{r} .
$$

Note that $\Psi^{\prime}$ for large $r$ is $\phi_{0}$ and does not depend on the location of the vortex line, i.e., it is independent of $\theta$. The required superposition is then

$$
\mathbf{A}_{T}^{\prime}=\sum_{i=1}^{N} \mathbf{A}_{1}^{\prime}=\sum_{i=1}^{N}\left(\mathbf{A}\left(r_{i}\right)-\frac{\hat{\boldsymbol{\theta}}_{i}}{r_{i}}\right)
$$

and

$$
\Psi_{T}^{\prime}=\phi_{0}+\sum_{i=1}^{N}\left[\phi\left(r_{i}\right)-\phi_{0}\right]=\phi_{T} .
$$

Because of the singular gauge transformation (2.4), $\mathbf{A}_{T}^{\prime}$ is not an acceptable physical solution, as it is singular at the center of each vortex line. This difficulty is removed by making a final gauge transformation on $\mathbf{A}_{T}^{\prime}$ and $\Psi_{T}^{\prime}$, namely,

$$
\mathbf{A}_{T}=\mathbf{A}_{T}^{\prime}+\sum_{i=1}^{N} \frac{\hat{\boldsymbol{\theta}}_{i}}{r_{i}}
$$


and

$$
\Psi_{T}=\exp \left(-i \sum \theta_{i}\right) \Psi_{T}
$$

This second singular gauge transformation produces a perfectly well-behaved $\boldsymbol{A}_{T}$.

The equations for the multivortex fields are

$$
\boldsymbol{\nabla} \times\left(\boldsymbol{\nabla} \times \mathbf{A}_{T}\right)+\left(\mathbf{A}_{T}-\boldsymbol{\Sigma} \frac{\hat{\boldsymbol{\theta}}_{i}}{r_{i}}\right) \phi_{T}{ }^{2}=0
$$

and

$$
-\nabla^{2} \phi_{T}+\left[\mathbf{A}_{T}-\Sigma \frac{\hat{\boldsymbol{\theta}}_{i}}{r_{i}}\right)^{2} \phi_{T}-\phi_{T}+K^{2} \phi_{T}{ }^{3}=0 .
$$

In the region of space far from a vortex (2.8) and (2.9) can be linearized with the following results:

$$
\sum_{i=1}^{N}\left[\nabla_{i} \times\left[\nabla_{i} \times \mathbf{A}\left(r_{i}\right)\right]-\left[\mathbf{A}\left(r_{i}\right)-\frac{\hat{\boldsymbol{\theta}}_{i}}{r_{i}}\right] \phi_{0}{ }^{2}\right]=0
$$

and

$$
\sum_{i=1}^{N}\left\{-\nabla_{i}^{2} \phi\left(r_{i}\right)+2\left[\phi\left(r_{i}\right)-\phi_{0}\right]\right\}=0 .
$$

These equations are clearly satisfied by $\mathbf{A}$ and $\phi$ in (2.7) as they are simply a superposition of the individual vortex asymptotic equations. On the other hand, if we are near a vortex, centered at $r_{1}$, for example,

$$
\begin{aligned}
& \boldsymbol{\nabla} \times\left(\boldsymbol{\nabla} \times \mathbf{A}_{T}\right) \rightarrow \nabla_{1} \times\left(\nabla_{1} \times \mathbf{A}_{1}\right) \\
&+ \text { exponentially small terms }, \\
& \mathbf{A}_{T}-\boldsymbol{\Sigma} \frac{\hat{\boldsymbol{\theta}}_{i}}{r} \rightarrow A_{1}-\frac{\hat{\boldsymbol{\theta}}_{1}}{r_{1}}+\text { exponentially small terms },
\end{aligned}
$$

and

$$
\phi_{T} \rightarrow \phi_{0}+\phi\left(r_{1}\right)-\phi_{0}+\text { exponentially small terms . }
$$

Therefore, near a vortex line, $\mathbf{A}_{T}$ and $\phi_{T}$ are nearly equal to the appropriate $\mathbf{A}$ and $\phi$ for that vortex line and will be asymptotically a solution to the Landau-Ginsberg equations when the vortex separation is large. This, of course, fails if there is an overlap region where the fields from two vortices are both large, that is, if the vortices are close together.

One word of caution is in order. The energy density can be written in a number of different forms by using partial integration and the field equations. For example, a term like $(A-1 / r)^{2} \phi^{2}$ can be written in terms of $(\boldsymbol{\nabla} \times \mathbf{A})^{2}$. It does not matter which form is used in the treatment of a single vortex. However, this is not true for the asymptotic multivortex solution. The $(\boldsymbol{\nabla} \times \mathbf{A})^{2}$ form is well behaved, while for any finite vortex separation $\phi$ is not exactly zero at $r=0$ and hence the first form will be infinite, in spite of the infinity having a small coefficient. For this reason one must be careful to write the energy density in a form that is not sensitive to the asymptotic nature of the solution.

We have used our asymptotic solution to calculate the potential energy between two like-sign vortex lines and find that an exponentially decreasing force exists. This force is repulsive in the type-II region $k>1 / \sqrt{2}$ and attractive in the type-I region. In addition to knowing the potential for large separation, we can use the energy of a vortex line with two units of flux to obtain the value of the potential for zero separation. In both type-I and type-II superconductors we find the value at the origin consistent with the sign of the long-range potential, in that, for type-II superconductors, an $n=2$ vortex has more than twice the energy of an $n=1$ vortex. This situation is reversed for type I. For $k=1 / \sqrt{2}$, the critical value, the long-range force, is found numerically to be zero to surprising accuracy. Subsequently, we have verified that this is indeed the correct behavior, since it is possible to show analytically that at the critical value the total energy depends only on the number of units of flux and is independent of the positions of the vortex lines. ${ }^{8}$

\section{SUPERPOSITION OF YANG-MILLS FLUX TUBES}

For the sake of completeness we will begin by reviewing the flux-tube solution of Ref. 5. In Ref. 5, the long-range properties of the Yang-Mills vacuum dielectric constant are used to construct a gauge-invariant effective action. This Lagrangian is written in terms of the electric vector potential $\mathrm{C}$, its associated scalar potential $C_{0}$, and the color vector fields $\mathbf{B}$ and $\mathbf{E}$ which play the role of auxiliary fields. The fields $\mathbf{B}$ and $\mathbf{E}$ reduce to the usual colormagnetic and -electric fields in the Abelian limit. In this language the Lagrangian is invariant under the magnetic gauge group which consists of the following transformations on the fields:

$$
\begin{aligned}
& C_{\mu} \rightarrow U^{-1} C_{\mu} U-\frac{i}{g} U^{-1} \partial_{\mu} U, \\
& \mathbf{B} \rightarrow U^{-1} \mathbf{B} U, \quad \mathbf{E} \rightarrow U^{-1} \mathbf{E} U,
\end{aligned}
$$

where $g$ is related to the usual Yang-Mills coupling constant $e$ via $e g=4 \pi$. The effective action contains a phenomenological "potential" $W(\mathbf{B}, \mathbf{E})$ which plays the role of the Higgs potential in the Abelian Higgs model discussed previously. The time-independent field equations obtained from the Lagrangian are

$$
\begin{aligned}
& D^{2} \mathbf{B}+\mathbf{D} C_{0}+\frac{\partial W}{\partial \mathbf{B}}, \\
& D^{2} \mathbf{E}+\nabla \times \mathbf{C}+i \mathbf{C} \times \mathbf{C}-\frac{\partial W}{\partial \mathbf{E}}=0, \\
& \mathbf{D}^{2} C_{0}-\mathbf{D} \cdot \mathbf{B}+i\left[D_{0} E_{j}, E_{j}\right]-i\left[D_{0} B_{j}, B_{j}\right]=0, \\
& \mathbf{D} \times(\nabla \times \mathbf{C})+D_{0} \mathbf{D} C_{0}-D_{0} \mathbf{B}-\mathbf{D} \times \mathbf{E}+i\left[\mathbf{D} E_{j}, E_{j}\right] \\
& \quad-i\left[\mathbf{D} B_{j}, B_{j}\right]+i \mathbf{D} \times(\mathbf{C} \times \mathbf{C})=0 .
\end{aligned}
$$

Here the gauge-covariant derivatives are the operators $\mathrm{D} F \equiv \nabla F+i[\mathbf{C}, F], D_{0} F \equiv i\left[C_{0}, F\right]$, and each field component is a matrix in color space, i.e., $C_{j}=\Sigma_{a} T_{a} C_{j}^{a}$ where the $T$ 's are the generators of the color group. In 
this case the color group is $\mathrm{SU}(2)$ and the T's are normalized so that $2 \operatorname{Tr}\left(T_{a} T_{b}\right)=\delta_{a b}$.

The color and space ansatz which works for a flux tube along the $z$ axis and which can be maintained in the course of superposition is that

$$
\begin{aligned}
& \mathbf{B}=\mathbf{B}_{1} T_{1}+\mathbf{B}_{2} T_{2}, \\
& \mathbf{E}=E T_{3} \widehat{\mathbf{e}}_{z}, \\
& \mathbf{C}=\mathbf{C} T_{3},
\end{aligned}
$$

and

$$
C_{0}=C_{1} T_{1}+C_{2} T_{2}
$$

Here $\mathbf{B}_{1}, \mathbf{B}_{2}$, and $\mathbf{C}$ lie in the $x-y$ plane. For the single flux-tube solution $\mathbf{C}$ and $\mathbf{B}_{2}$ are in the $\hat{\boldsymbol{\theta}}$ direction, $\mathbf{B}_{1}$ is in the $\hat{\mathbf{r}}$ direction, and $C_{0}$ has only a one component of color. The asymptotic behavior of the solution resembles that of a superconducting vortex line with $\mathbf{C} \rightarrow \hat{\boldsymbol{\theta}} / r$, $\mathbf{B}_{1} \rightarrow b \hat{\mathbf{r}}$, and $\mathbf{B}_{2} \rightarrow-b \hat{\boldsymbol{\theta}}$, where $b$ is a constant determined from the "potential" $W$. All other fields vanish exponentially.

The gauge transformations required to construct the multi-flux-tube solution are rotations about the 3 -axis in color space. For our color ansatz, these SU(2) transformations can be most easily represented as $U(1)$ gauge transformations on the complex fields

$$
\mathbf{B}_{+}=\mathbf{B}_{1}+i \mathbf{B}_{2}
$$

and

$$
C_{+}=C_{1}+i C_{2} .
$$

Thus the gauge transformation is

$$
\mathbf{B}^{\prime}=U^{-1} \mathbf{B} U, C_{0}^{\prime}=U^{-1} C_{0} U
$$

and

$$
\mathbf{C}^{\prime}=\mathbf{C}-i U^{-1} \nabla U
$$

where $U=e^{-i \theta T_{3}}$ is identical to the $U(1)$ gauge transformation

$$
\mathbf{B}_{+}^{\prime}=e^{-i \theta} \boldsymbol{B}_{+}, C_{+}^{\prime}=e^{-i \theta} C_{+}, \text {and } \mathbf{C}^{\prime}=\mathbf{C}-\frac{\hat{\boldsymbol{\theta}}}{r} .
$$

Here the C's are to be identified as the color 3component of the vector potential and the real (imaginary) parts of $B_{+}$and $C_{+}$are the color-1 (-2) components, respectively, of the corresponding fields. Having reduced the gauge group to that of the vortex line case, we proceed exactly as before. The gauge transformation needed to produce a simple asymptotic behavior is, in fact, the one given above. Clearly $\mathbf{C}^{\prime}$ will vanish exponentially and

$$
\mathbf{B}_{+}^{\prime}=e^{i \theta} \mathbf{B}_{+} \rightarrow b e^{-i \theta}(\widehat{\mathbf{r}}-i \widehat{\boldsymbol{\theta}})=b\left(\widehat{\mathbf{e}}_{x}-i \widehat{\mathbf{e}}_{y}\right)=\mathbf{B}_{0}
$$

As in the superconductivity case, the gauge transformation that removes the $1 / r$ term from the vector potential also automatically produces a constant behavior for $\mathbf{B}$ which is independent of the location of the center of the flux tube. We now superimpose the solutions to obtain

$$
\begin{aligned}
& \mathbf{C}_{T}^{\prime}=\sum \mathbf{C}^{\prime}\left(\mathbf{r}_{i}\right)=\sum\left[\mathbf{C}\left(\mathbf{r}_{i}\right)-\frac{\hat{\boldsymbol{\theta}}_{i}}{r_{i}}\right], \\
& \mathbf{E}_{T}^{\prime}=\mathbf{E}_{T}=\boldsymbol{\Sigma} \mathbf{E}\left(\mathbf{r}_{i}\right), \\
& \mathbf{B}_{+T}^{\prime}=b\left(\widehat{\mathbf{e}}_{x}-i \widehat{\mathbf{e}}_{y}\right)+\Sigma e^{-i \theta_{i}} \mathbf{b}_{+}\left(\mathbf{r}_{i}\right), \\
& C_{+T}^{\prime}=\sum e^{-i \theta_{i}} \mathbf{C}_{+}\left(r_{i}\right),
\end{aligned}
$$

where

$$
\mathbf{b}_{+}\left(\mathbf{r}_{i}\right)=\mathbf{B}_{+}\left(\mathbf{r}_{i}\right)-e^{i \theta_{i}} b\left(\widehat{\mathbf{e}}_{x}-i \widehat{\mathbf{e}}_{y}\right) .
$$

The unacceptable singularities present in these fields are removed by the overall gauge transformation $U=\exp \left(i \Sigma_{j} \theta_{j}\right)$. The resulting multi-flux-tube fields are

$$
\begin{aligned}
& \mathbf{C}_{T}=\boldsymbol{\Sigma} \mathbf{C}\left(\mathbf{r}_{i}\right), \\
& \mathbf{B}_{+T}=b\left(\hat{\mathbf{e}}_{\boldsymbol{x}}-i \widehat{\mathbf{e}}_{\boldsymbol{y}}\right) U+\sum U_{i} \mathbf{b}_{+}\left(\mathbf{r}_{i}\right),
\end{aligned}
$$

and

$$
C_{+T}=\sum U_{i} C_{+}\left(\mathbf{r}_{i}\right)
$$

where $U_{i}=e^{-i \theta_{i}} U$. Thus the vector potential is a simple superposition of the individual flux-tube potentials, while $B$ and $C_{0}$ are a superposition of single flux-tube fields, but each is gauge transformed in a manner determined by the positions of the other flux tubes.

To prove that this is in fact an asymptotic solution we must first show that for $\mathbf{r}$ far from the center of any flux tube, the leading exponential behavior satisfies the field equations. The second step is to show that in the region of space near a flux tube both the fields and the field equations are approximately (inaccurate only to exponentials in the separation of neighboring flux tubes) those of the single flux tube. We begin this process by first investigating the various ways that $\mathbf{C}$ appears in the field equations (3.2)-(3.5). We first note that all $\mathbf{C} \times \mathbf{C}$ terms vanish because $\mathbf{C}$ is in a single color direction, and that $\boldsymbol{\nabla} \times \mathbf{C}$ behaves exponentially since the $1 / r$ terms do not contribute. The only other terms containing $\mathbf{C}$ are through $\mathbf{D}$ operating on $\mathbf{B}$ or $C_{0}$ (recall that $\mathbf{E}$ and $\mathbf{C}$ are in the same color direction). Consider the action of $\mathbf{D}$ on a particular term in $C_{+T}$ (the complex notation simplifies the algebra in that $\mathbf{D} \equiv \boldsymbol{\nabla}-i \mathbf{C})$ :

$$
\begin{aligned}
\mathrm{D} C_{+i}= & \mathrm{D} U_{i} C_{0}\left(r_{i}\right) \\
=U_{i}[ & {\left[\nabla-i \mathbf{C}\left(\mathbf{r}_{i}\right)\right.} \\
& \left.-i \sum_{j \neq i}\left[\mathbf{C}\left(r_{j}\right)-\frac{\widehat{\theta}_{j}}{r_{j}}\right]\right] C_{0}\left(\mathbf{r}_{i}\right) .
\end{aligned}
$$

The result of commuting $U_{i}$ through $\mathbf{D}$ is to produce the correct $\mathbf{D}_{i}$ for a single flux tube centered at $r_{i}=0$ plus extra terms which are exponentially small. For large $r_{i}$ these terms can be dropped because $C_{0}$ is already exponentially small, and the product becomes a secondorder term. On the other hand, if $r_{i}$ is small, $\mathbf{C}\left(r_{i}\right)$ is large and the extra small terms can be neglected. The result is that 


$$
\mathrm{D} C_{+i} \simeq U_{i} \mathrm{D}_{i} C_{0}\left(r_{i}\right)
$$

The effect of commuting $U_{i}$ through $\mathbf{D}$ is exactly the same for an individual flux-tube term in $B_{+T}$, i.e.,

$$
\mathbf{D} U_{i} \mathbf{b}_{+}\left(\mathbf{r}_{i}\right) \simeq U_{i} \mathbf{D}_{i} \mathbf{b}_{+}\left(\mathbf{r}_{i}\right) .
$$

Let us now investigate the behavior of the constant term in $\mathbf{B}_{+T}$ :

$$
\mathbf{D U b}\left(\widehat{\mathbf{e}}_{\boldsymbol{x}}-i \widehat{\mathbf{e}}_{\boldsymbol{y}}\right)=-i U\left(\boldsymbol{\Sigma} \mathbf{C}\left(r_{i}\right)-\frac{\theta_{i}}{r_{i}}\right) b\left(\widehat{\mathbf{e}}_{x}-i \widehat{\mathbf{e}}_{y}\right)
$$

If we associate each of these $N$ terms with the $N$ terms of the form given in Eq. (3.23) we obtain

$$
\begin{aligned}
-i U\left[\mathbf{C}\left(\mathbf{r}_{i}\right)-\frac{\hat{\boldsymbol{\theta}}_{i}}{r}\right] b\left(\hat{\mathbf{e}}_{x}-i \widehat{\mathbf{e}}_{y}\right)+U_{i} \mathbf{D}_{i} \mathbf{b}_{+}\left(\mathbf{r}_{i}\right) & =+U_{i}\left(\boldsymbol{\nabla}-i \mathbf{C}\left(\mathbf{r}_{i}\right)\right) b e^{i \theta_{i}}\left(\widehat{\mathbf{e}}_{x}-i \widehat{\mathbf{e}}_{y}\right)+U_{i} \mathbf{D}_{i} \mathbf{b}_{+}\left(\mathbf{r}_{i}\right) \\
& =U_{i} \mathbf{D}_{i} \mathbf{B}\left(\mathbf{r}_{i}\right) .
\end{aligned}
$$

From these observations it is clear that substituting the multi-flux-tube ansatz into any of the field equations (3.2)-(3.5) results in a sum of individual flux-tube equations, each with a different phase in the complex notation or a different $S U(2)$ transformation in the matrix notation. However, because the individual equations are satisfied, the multi-flux-tube fields are solutions.

We have used these asymptotic solutions to calculate the interaction energy of two parallel flux tubes. Figure 1 shows the energy as a function of separation for two like-sign flux tubes for a particular solution [solution (d) in Ref. 5]. The interaction is repulsive at long range with the expected exponential dependence. The decrease observed at small $r$ is what we expect to happen. As in the case of two superconducting vortex lines, the energy of a flux tube with two units of flux can be used to determine the potential for zero separation. In this case, the gauge group is SU(2) and on topological grounds one can show that this state is equivalent to the vacuum. Therefore the net interaction energy at zero separation must be zero.

To see explicitly that a flux tube with two units of flux is equivalent to the vacuum, we proceed as follows. The vector potential for a two-flux tube is a solution to our equations which is well behaved as $r \rightarrow 0$ and for which $\mathbf{C} \rightarrow\left(2 T_{3} / r\right) \hat{\boldsymbol{\theta}}$ for large $r$. An example of such a solution is

$$
\begin{aligned}
& \mathbf{C}=2\left(T_{1} \cos \theta+T_{2} \sin \theta\right) \frac{\hat{\mathbf{r}}}{1+r^{2}}+2\left(-T_{1} \sin \theta+T_{2} \cos \theta\right) \frac{\widehat{\boldsymbol{\theta}}}{1+r^{2}}+2 \frac{T_{3} r \hat{\boldsymbol{\theta}}}{1+r^{2}}, \\
& \mathbf{B}=\frac{b}{1+r^{2}}\left\{\hat{\mathbf{e}}_{x}\left[\left(1+r^{2} \cos 2 \theta\right) T_{1}+r^{2} \sin 2 \theta T_{2}-2 r \sin \theta T_{3}\right]-\hat{\mathbf{e}}_{y}\left[\left(1-r^{2} \cos 2 \theta\right) T_{2}+r^{2} \sin 2 \theta T_{1}+2 r \cos \theta T_{3}\right]\right\} .
\end{aligned}
$$

Here $\mathbf{E}$ and $C_{0}$ are both zero. For large $r$

$$
\begin{aligned}
\mathbf{C} \rightarrow & \frac{2 T_{3} \hat{\boldsymbol{\theta}}}{r}, \\
\mathbf{B} \rightarrow b[ & {\left[\hat{\mathbf{e}}_{x}\left(\cos 2 \theta T_{1}+\sin 2 \theta T_{2}\right)\right.} \\
& \left.+\widehat{\mathbf{e}}_{y}\left(\cos 2 \theta T_{2}-\sin 2 \theta T_{1}\right)\right] .
\end{aligned}
$$

That this is a solution to the field equations follows from the fact that $\mathbf{C}$ and $\mathbf{B}$ are the result of a simple gauge transformation of the vacuum state:

$$
\begin{aligned}
& \mathbf{C}=0, \\
& \mathbf{B}=b\left(\widehat{\mathbf{e}}_{x} T_{1}-\widehat{\mathbf{e}}_{y} T_{2}\right) .
\end{aligned}
$$

The nonsingular gauge transformation connecting the fields of Eqs. (3.26) and (3.27) to the vacuum fields (3.30) and $(3.31)$ is

$$
U=\exp \left[2 i\left(\cos \theta T_{1}+\sin \theta T_{2}\right) \arctan r\right]
$$

It follows that the total energy for the fields in Eqs. (3.26) and (3.27) is that of the vacuum, i.e., zero.
In order to investigate the interaction between opposite-sign flux tubes we must first construct an antiflux-tube solution. Simply rotating the $z$ axis of a flux tube into the negative $z$ axis fails, as this changes the asymptotic vacuum state. For the method of superposi-

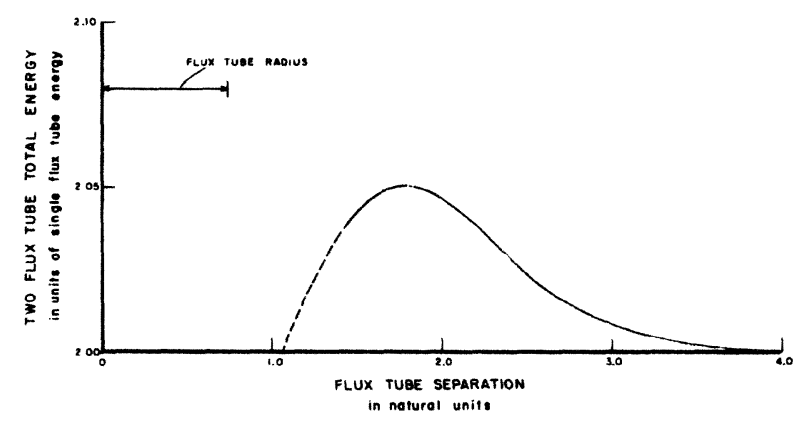

FIG. 1. The total energy per unit length of two flux tubes as a function of separation. The dashed portion of the curve is the guessed behavior required to reach the correct value of zero at zero separation. The flux-tube solution used is that of solution (d) of Ref. 5. Other solutions have the same qualitative behavior. 
tion to work the individual solutions must asymptotically approach the same $\mathbf{B}_{0}$. Our procedure will be to construct a general nonsingular $\mathrm{SU}(2)$ gauge transformation that changes the flux by two units but leaves $\mathbf{B}_{0}$ unchanged.
This will show that all flux tubes with odd units of flux are gauge equivalent and allow us to obtain the anti-fluxtube solution needed for the superposition.

A gauge transformation with the required properties is

$$
U=\frac{1}{1+f^{2}(r)}\left[1-f(r)^{2} \cos \theta+2 i f(r)(\cos \theta+\cos 2 \theta) T_{1}+2 i f(r)(\sin \theta+\sin 2 \theta) T_{2}+2 i f^{2}(r) \sin \theta T_{3}\right],
$$

where $f(r)$ is any function which vanishes as $r \rightarrow 0$ and blows up as $r \rightarrow \infty$. A suitable $f(r)$ for our purposes is

$$
f(r)=e^{r / r_{0}}-1 \text {, }
$$

where $r_{0}$ sets the scale of the gauge transformation. We can see that $U$ changes the flux by two units by looking at the large- $r$ behavior of $U$. For $r \gg r_{0}$

$$
U \rightarrow-\cos \theta+2 i \sin \theta T_{3}=e^{-2 i \theta T_{3}} .
$$

The resulting electric vector potential is

$$
\mathbf{C}^{\prime}=\mathrm{C}-\frac{2 T_{3}}{r} \hat{\boldsymbol{\theta}}
$$

and the flux has been reduced by two units. This result for large $r$ is the same as that obtained by using the singular gauge transformation $e^{-2 i \theta T_{3}}$; however $U$ is not a singular gauge transformation.

The anti-flux-tube fields are obtained by applying $U$ to the flux-tube fields. For convenience in the construction of the flux-tube-anti-flux-tube solution we will choose the arbitrary parameter $r_{0}$ to be much smaller than the size of a flux tube so that the asymptotic form for $U$ can be used. This allows us to return to the complex notation with $U$ being

$$
U=e^{-2 i \theta} \text {. }
$$

The superposition of a flux tube at $r_{1}=0$ and anti-fluxtube at $r_{2}=0$ is

$$
\begin{aligned}
& \mathbf{C}_{T}=\mathbf{C}\left(r_{1}\right)+\mathbf{C}\left(r_{2}\right)-\frac{2}{r_{2}} \hat{\boldsymbol{\theta}}_{2}, \\
& \mathbf{B}_{T}=\mathbf{B}_{0} e^{i \theta_{1}-i \theta_{2}}+e^{-i \theta_{2}} \mathbf{b}\left(r_{1}\right)+\mathbf{b}\left(r_{2}\right) e^{i \theta_{1}-2 i \theta_{2}}, \\
& \mathbf{E}_{T}=\mathbf{E}\left(r_{1}\right)+\mathbf{E}\left(r_{2}\right), \\
& C_{0 T}=C_{0}\left(r_{1}\right) e^{i \theta_{2}}+C_{0}\left(r_{2}\right) e^{i \theta_{1}-2 i \theta_{2}},
\end{aligned}
$$

where all functions are those for the flux-tube solution. The interaction energy is exactly the same as the energy of two flux tubes, since the above fields are the same as those one gets by applying the gauge transformation $U$ to the two flux-tube solutions. Therefore, the only possible energy difference in the flux-tube-anti-flux-tube case comes from the portion of the volume integral near the center of the anti-flux-tube. However, because $U$ is a nonsingular gauge transformation, no fields are large in this volume and as $r_{0}$ is made very small, this region contributes a negligible amount to the interaction energy. Therefore the flux-tube-anti-flux-tube interaction energy is identical to that of two flux tubes. It should be noted that this behavior is obtained because of the existence of a nonsingular SU(2) gauge transformation connecting the flux tube and the anti-flux-tube. This behavior is very different from the case of superconductivity where the vortex-antivortex interaction is always attractive and not related to the two-vortex interaction.

As a result of this behavior, a potential barrier exists between flux tubes and anti-flux-tubes, preventing their annihilating each other. This result may have several interesting consequences. The first is that a toroidal flux ring with a diameter of the order of the radius of the repulsive region of the potential should be metastable. Crude estimates of the energy associated with such a state indicate that its mass could be comparable to the glueball mass obtained in Ref. 7 for the same set of parameters. The second is a possible application to nuclear physics. A simple QCD model for a nucleon is a single flux tube connecting a diquark system to a single quark. Consider, for example, the deuteron, which would be composed of two such flux tubes. If the flux tubes are nearly parallel, the flux-tube potential provides a flavor-independent longrange repulsive contribution to the nuclear force to be added to the usual meson-exchange terms.

\section{MULTIGLUEBALL SOLUTIONS}

We next wish to employ the general method described in Sec. II to superimpose the glueball solutions of Ref. 7. In the previous cases, using the Abelian gauge group $U(1)$ was sufficient to construct the multisoliton solutions. In the case of spherically symmetric solutions the color and space structure is more complicated, necessitating the use of more general SU(2) transformations.

We begin by briefly reviewing the glueball solution obtained in Ref. 7. The field ansatz used is

$$
\begin{aligned}
& C_{0}=C_{0}(r) \hat{\mathbf{r}} \cdot \mathbf{T}, \\
& \mathbf{C}=C_{1}(r) \hat{\mathbf{r}} \times \mathbf{T}, \\
& \mathbf{E}=-E_{2}(r) \hat{\mathbf{r}} \times(\hat{\mathbf{r}} \times \mathbf{T})+E_{3}(r) \hat{\mathbf{r}}(\hat{\mathbf{r}} \cdot \mathbf{T}), \\
& \mathbf{B}=-B_{2}(r) \hat{\mathbf{r}} \times(\hat{\mathbf{r}} \times \mathbf{T})+B_{3}(r) \hat{\mathbf{r}}(\hat{\mathbf{r}} \cdot \mathbf{T}) .
\end{aligned}
$$

The solution has the asymptotic behavior

$$
\begin{aligned}
& \mathbf{C} \rightarrow-\frac{2}{r} \hat{\mathbf{r}} \times \mathbf{T}, \\
& \mathbf{B} \rightarrow b(\mathbf{T}-2 \hat{\mathbf{r}} \hat{\mathbf{r}} \cdot \mathbf{T}), \\
& \mathbf{E} \rightarrow 0,
\end{aligned}
$$




$$
C_{0} \rightarrow 0 \text {. }
$$

As before, the vector potential is proportional to $1 / r$ and $B$ goes to a constant. The gauge transformation necessary to make $\mathbf{C}$ vanish exponentially is

$$
U=2 \hat{\mathbf{r}} \cdot \mathbf{T}=-i e^{\hat{\mathbf{r}} \cdot \mathbf{T} \pi / 2},
$$

which has the properties that

$$
U^{-1} \hat{\mathbf{r}} \times \mathbf{T} U=-\hat{\mathbf{r}} \times \mathbf{T}
$$

and

$$
-i U^{-1} \nabla \mathbf{U}=\frac{2}{r} \hat{\mathbf{r}} \times \mathbf{T} .
$$

The gauge transformed asymptotic $\mathbf{B}$ is

$$
\mathbf{B}^{\prime}=2 \hat{\mathbf{r}} \cdot \mathbf{T} b(\mathbf{T}-2 \hat{\mathbf{r}} \hat{\mathbf{r}} \cdot T) 2 \hat{\mathbf{r}} \cdot \mathbf{T}=b \mathbf{T},
$$

which again is independent of the location of the glueball.

The superposition then yields

$$
\begin{aligned}
& \mathbf{C}_{T}^{\prime}=-\sum_{i}\left(C_{1}\left(r_{i}\right)+\frac{2}{r_{i}}\right) \widehat{\mathbf{r}}_{i} \times \mathbf{T}, \\
& \mathbf{B}_{T}^{\prime}=b \mathbf{T}+\sum_{i} U_{i} \mathbf{b}_{i} U_{i},
\end{aligned}
$$

$$
\begin{aligned}
& C_{0 T}=\sum_{i} C_{0}\left(r_{i}\right) \hat{\mathbf{r}}_{i} \cdot \mathbf{T}, \\
& \mathbf{E}_{T}=\sum_{i} U_{i} \mathbf{E}\left(\mathbf{r}_{i}\right) U_{i},
\end{aligned}
$$

where

$$
\mathbf{b}_{i}=\mathbf{B}\left(r_{i}\right)-b\left(\mathbf{T}-2 \hat{\mathbf{r}}_{i} \hat{\mathbf{r}}_{i} \cdot \mathbf{T}\right)
$$

and

$$
U_{i}=2 \widehat{\mathbf{r}}_{i} \cdot \mathbf{T} .
$$

The final step is to use an overall gauge transformation to remove the singularities in $\mathbf{C}_{T}^{\prime}$. Clearly, the gauge transformation needed for the $i$ th term in $\mathbf{C}_{T}^{\prime}$ is a matrix which has $U_{i}$ as a factor on the left and introduces no new singularities. Because the different $U$ 's do not commute this is difficult to achieve. At present we have onily explicitly solved the problem for the case of two glueballs, but we believe a general solution is possible.

In the case of two glueballs located on the $z$ axis, the gauge transformation that behaves like the Abelian transformation of the previous sections is

$$
U=2 U_{1} T_{3} U_{2}=2 U_{2} T_{3} U_{1} \text {. }
$$

The resulting vector potential is

$\mathbf{C}_{T}=U^{-1} \mathbf{C}_{T}^{\prime} U-i U^{-1} \nabla U=4 U_{2} T_{3} \hat{\mathbf{r}}_{1} \times \mathbf{T}\left(C_{1}\left(r_{1}\right)+\frac{2}{r_{1}}\right) T_{3} U_{2}+4 U_{1} T_{3} \widehat{\mathbf{r}}_{2} \times \mathbf{T}\left(C_{1}\left(r_{2}\right)+\frac{2}{r_{2}}\right) T_{3} U_{1}-i U^{-1} \nabla \mathbf{U}$

Because of the symmetry of $U^{-1}, U^{-1} \nabla U$ can be written in two forms:

$$
\begin{aligned}
-i U^{-1} \nabla U & =-8 U_{2} T_{3} \frac{\hat{\mathbf{r}}_{1} \times \mathbf{T}}{r_{1}} T_{3} U_{2}-2 \frac{\hat{\mathbf{r}}_{2} \times \mathbf{T}}{r_{2}} \\
& =-8 U_{1} T_{3} \hat{\mathbf{r}}_{2} \times \mathbf{T} T_{3} U_{1}-2 \frac{\hat{\mathbf{r}}_{1} \times \mathbf{T}}{r_{2}}
\end{aligned}
$$

When the first form is used in Eq. (4.18), it is clear that $\mathbf{C}_{T}$ is regular as $r_{1} \rightarrow 0$ because the singularities cancel, but the behavior as $r_{2} \rightarrow 0$ is not obvious. Conversely, if the second form is used, the singularity as $r_{2} \rightarrow 0$ is removed. One concludes that all singularities are removed.

All other fields transform according to the homogeneous gauge transformation

$$
C_{0 T}=4 U_{2} T_{3} C_{0}\left(r_{1}\right) \hat{\mathbf{r}}_{1} \cdot \mathbf{T} T_{3} U_{2}+4 U_{1} T_{3} C_{0}\left(r_{2}\right) \hat{\mathbf{r}}_{2} \cdot \mathbf{T} T_{3} U_{1},
$$

$\mathbf{B}_{T}=U b \mathrm{~T} U+4 U_{2} T_{3} \mathrm{~b}_{1} T_{3} U_{2}+4 U_{1} T_{3} \mathrm{~b}_{2} T_{3} U_{1}$,

and

$$
\mathbf{E}_{T}=4 U_{2} T_{3} \mathbf{E}\left(r_{1}\right) T_{3} U_{2}+4 U_{1} T_{3} \mathbf{E}\left(r_{2}\right) T_{3} U_{1} .
$$

As in the previous cases, the individual glueball terms in the field equations are the original fields changed by a gauge transformation dependent on the position of the other glueball. Because of the symmetry of $U$, the constant term in $\mathbf{B}_{T}$ can be combined with the $\mathbf{b}$ term of either glueball to form the correct total $\mathbf{B}$ for that glueball.

The demonstration that these fields satisfy Eqs. (3.2) $-(3.5)$ is exactly as before. Commuting $U_{i} T_{3}$ through the $\mathbf{D}$ operators produces the necessary $\mathbf{D}_{i}$ for that glueball. The resulting equations are a sum of the original single glueball equations sandwiched between color matrices and since the original single glueball equations are equal to zero, the multiglueball fields are asymptotic solutions.

The two-glueball interaction energy falls exponentially with distance and no long-range Van der Waals forces exist.

\section{CONCLUSIONS}

We have developed a general method for superimposing gauge soliton solutions to obtain an asymptotic multisoliton solution. This has been possible in spite of highly nonlinear field equations and the fact that the fields go to constant values, or like $1 / r$, at large distances.

The first conclusion is that, in spite of the fact that the basic long-range interaction in QCD is a linear potential between colored sources, the potential between colorless localized objects is not long range, and in fact falls exponentially with separation. We have explicitly shown 
this to be the case for the interaction between flux tubes and between glueballs, but we expect the same result to apply between hadrons (baglike objects containing quarks) because the underlying long-range force is the same.

We have also found that the interaction between two flux tubes has a barrier outside of an attractive well. Therefore flux tubes are inhibited from annihilating each other, so that a toroidal flux ring is probably metastable. Such a structure could well be a glueball; our numerical estimates suggest the mass of this type of glueball is about the same, or perhaps even lower, than that of the spherical glueball of Ref. 7.

The barrier has other phenomenological implications for particle production in high-energy collisions. If superconducting analogies hold, then hadronization results from the breaking up of an expanding region of a colorelectric field into electric flux tubes when the contained electric field has fallen to the critical value. The barrier in the interaction between these tubes then determines the rates at which they annihilate and consequently how many of them will survive as hadrons after the electric field has fully dissipated and the flux tubes, or bags, have separated.

In other versions of production, the original configuration is itself a flux tube, which repeatedly splits into smaller tubes as quark-antiquark pairs are formed. Again the interaction between these tubes influences the fixed hadronic multiplicities and rapidity distribution.

Our conclusions that $\mathrm{SU}(2)$ flux and anti-flux-tubes have a net attractive force is certainly more general than our detailed calculation for these solitons as it follows from the gauge equivalence between the vacuum and all flux tubes with even units of flux.

In QCD the gauge group is $\mathrm{SU}(3)$, therefore in this case there are two gauge-independent solitons, those with one and two units of color-electric flux. Although we do not have the solutions for these solitons we can still determine their net (average) interaction.

First we expect a flux tube to have the same self-energy as its anti-flux-tube. However the one-unit anti-flux-tube is gauge equivalent to the flux tube with two units of flux. This follows from the fact that all flux tubes differing by three units of flux are gauge equivalent and that flux tubes with $3 N$ units are equivalent to the vacuum state. Therefore we expect flux tubes with one and two units of flux to have the same self-energy. Thus the potential between two identical flux tubes must fall from $2 E_{0}$ at large separation ( $E_{0}$ self-energy of a flux tube) to $E_{0}$ at the origin indicating a net attractive force. The potential between a flux-one tube and a flux-two tube falls to zero at the origin, hence indicating a stronger net attraction. The interaction between a flux tube and an anti-flux-tube is the same as that between flux one and flux two because of the gauge equivalence discussed above.

In the glueball case we have answered the question as to whether more than one glueball can exist for our model of long-range Yang-Mills theory. Unfortunately, our result is not as general as we would like, only reaching the twoglueball level. It seems certain that the gauge transformation necessary for the general case can be constructed, but it may involve path-ordered color operators which cannot be expressed simply for more than two glueballs.

\section{ACKNOWLEDGMENTS}

One of us (J.S.B.) would like to thank the Argonne National Laboratory High Energy Theory group for its hospitality during part of this work. One of the authors (J.S.B.) was supported in part by National Science Foundation Grant No. PHY 84-05648. Another (F.Z.) was supported in part by the U.S. Department of Energy under Contract No. DEAC 03-81-ER 40050.
'See, for example, C. DeTar and J. Donoghue, Annu. Rev. Nucl. Sci. 33, 235 (1983).

${ }^{2}$ S. Adler, Phys. Lett. 110B, 302 (1982).

${ }^{3}$ G. 't Hooft, Nucl. Phys. B153, 141 (1979); S. Mandelstam, Phys. Rev. D 19, 302 (1982); C. Rosenzweig and V. P. Nair, Phys. Lett. 135B, 450 (1984).

${ }^{4}$ H. B. Nielsen and P. Olesen, Nucl. Phys. B61, 45 (1973).

${ }^{5}$ M. Baker, J. S. Ball, and F. Zachariasen, Phys. Rev. D 31, 2575 (1985). The coupling constant has been scaled out of these equations.

6D. Saint-James, G. Sarma, and E. J. Thomas, Type II Superconductivity (Pergamon, New York, 1969).

${ }^{7}$ M. Baker, J. S. Ball, F. Z. Chen, and F. Zachariasen, Phys.
Rev. D 33, 1415 (1986).

${ }^{8}$ What has been shown is that at the critical value of $k$, the energy of any two-dimensional solution to the Landau-Ginsberg equations is proportional to the total flux. This means that the energy cannot depend on the location of vortex lines but only on the total number. This result, which is more general than the well-known result that the energy of a vortex with $N$ units of flux is $N$ times that of the $N=1$ vortex, appears to be known to workers in the field. Unfortunately, we have not been able to find a reference containing this result but only statements to the effect that it is in fact known. See, for example, L. Kramers, Phys. Lett. 23, 619 (1966). 